\title{
Four-colour photometry of eclipsing binaries
}

\author{
XXXIX. Light curves of the pre-main sequence triple system TY Coronae Australis ${ }^{\star, \star \star}$ \\ L.P.R. Vaz ${ }^{1,3}$, J. Andersen ${ }^{2}$, B.W. Casey $^{3}$, J.V. Clausen ${ }^{2}$, R.D. Mathieu ${ }^{3}$, and I. Heyer ${ }^{4}$ \\ 1 Departamento de Física, ICEx, Universidade Federal de Minas Gerais, C.P. 702, 30.123-970 - Belo Horizonte, MG, Brazil \\ 2 Niels Bohr Institute for Astronomy, Physics and Geophysics; Astronomical Observatory, Juliane Maries Vej 30, DK-2100 \\ Copenhagen, Denmark \\ 3 Department of Astronomy, University of Wisconsin, Madison, Wisconsin 53706, U.S.A. \\ ${ }^{4}$ Space Telescope Science Institute, 3700 San Martin Drive, Baltimore, MD 21218, U.S.A.
}

Received July 3; accepted November 28, 1997

\begin{abstract}
Complete uvby light curves of the detached Herbig Be eclipsing binary TY Coronae Australis are presented. A total of 1789 photometric measurements in each of the four colours were obtained in 1989 and in 1992-1994. A detailed analysis of the $y$ light curve obtained in 19921993 is published separately (Casey et al. 1997). The reflection nebula around the system contributes about $30 \%$ of the light in all four passbands. Here we present and discuss the non-eclipse-related photometric variability of the system. We suggest that these variations are the result of variable obscuration, possibly linked to dust shells physically associated to the system.
\end{abstract}

Key words: binaries: eclipsing: — stars: individual: TY CrA - stars: pre-main sequence

\section{Introduction}

The eclipsing nature of the Herbig Be star TY CrA was discovered by Kardopolov et al. (1981) from photoelectric observations. Later photometry (e.g. Vitrichenko \& Shevchenko 1995) and spectroscopy (Casey et al. 1993, hereinafter Paper I; Lagrange et al. 1993; Corporon et al. 1994; Casey et al. 1995, Paper II) have shown that the orbit is circular with a period of $P=2$ d 888779 . High S/N spectra show lines of both eclipsing components and in

Send offprint requests to: L.P.R. Vaz at address 1

* Based on observations collected with the Danish $50 \mathrm{~cm}$ Strömgren Automatic Telescope, SAT, at the European Southern Observatory (ESO), La Silla, Chile.

$\star \star$ Tables $2 \mathrm{a}-2 \mathrm{~d}$ will only be available in electronic form at the CDS via anonymous ftp 130.79.128.7 or http://cdsweb.ustrasbg.fr/Abstract.html addition lines of a tertiary star in a longer period orbit (Corporon et al. 1994; Paper II; Corporon et al. 1996). An outstanding peculiarity of TY CrA is the very narrow spectral lines of the primary star, indicating a $v \sin i$ of less than $10 \mathrm{~km} / \mathrm{s}$ (Paper I; Lagrange et al. 1993). Thus the primary appears virtually non-rotating despite its location in a young, short-period binary system with an already circularized orbit (see also discussion in Beust et al. 1997 and Casey et al. 1997, Paper III).

TY CrA is embedded in the densest part of the R CrA molecular cloud (Harju et al. 1993) in the reflection nebula NGC 6726/7. It forms a pair with HD 176386 (Sep. 1!1), which is a visual binary (Sep. $4^{\prime \prime} .1$ ) and has occasionally been confused with TY CrA (e.g. Proust et al. 1981). The location, reflection nebula, and strong infrared excess of TY CrA are all evidence that it is still very young. After EK Cep (Popper 1987), TY CrA is only the second doublelined eclipsing binary which includes a pre-main sequence star, and as such is of particular interest.

The cloud associated with TY CrA is very peculiar: its infrared emission (IRAS point source 18583-3657) peaks beyond $100 \mu \mathrm{m}$, suggesting very cold dust as its origin (Friedemann et al. 1996). The densest clump of the CrA cloud is one of the coldest ever measured (an upper limit of $8 \mathrm{~K}$, according to Harju et al. 1993 and Haikala 1994), which should favour the formation of multiple stars (Durisen \& Sterzik 1994). In fact, TY Cra is a triple system, located at a projected distance of $8500 \mathrm{AU}$ from the visual binary HD 176386 , the companion of which is very red (private communication from Dr. H. Zinnecker from a $10 \mu \mathrm{m}$ exposure obtained with TIMMI and the ESO $3.6 \mathrm{~m}$ telescope). Practically all stars associated with the $\mathrm{R}$ CrA region are very young (Bibo et al. 1992) and we would expect that most of them are multiple.

In order to establish the precise properties and evolutionary stages of the stars in TY CrA, gain insight in 
Table 1. Data for TY CrA and the comparison stars. The uvby indices for TY CrA correspond to phase 0.349 (1994), while its $\beta$ index was obtained at 0.625 (1993)

\begin{tabular}{|c|c|c|c|}
\hline & TY CrA & Comparison 1 & Com \\
\hline HD & - & 176423 & 176497 \\
\hline $\mathrm{SAO}$ & 210829 & 210834 & 210840 \\
\hline CPD & $-37^{\circ} 13024$ & $-36^{\circ} 13235$ & $-36^{\circ} 13242$ \\
\hline$\alpha_{2000}$ & $18^{\mathrm{h}} 58^{\mathrm{m}} 19^{\mathrm{s}}$ & $18^{\mathrm{h}} 58^{\mathrm{m}} 28^{\mathrm{s}}$ & $18^{\mathrm{h}} 58^{\mathrm{m}} 45^{\mathrm{s}}$ \\
\hline$\delta_{2000}$ & $-36^{\circ} 56^{\prime} 52^{\prime \prime}$ & $-36^{\circ} 44^{\prime} 12^{\prime \prime}$ & $-36^{\circ} 36^{\prime} 02^{\prime \prime}$ \\
\hline Sp. type & B8-B9: & A1 III-IV & A0 IV \\
\hline$V$ & $\begin{array}{r}9.299 \\
\pm 7\end{array}$ & $\begin{array}{r}9.017 \\
\pm 6\end{array}$ & $\begin{array}{r}8.377 \\
\pm 8\end{array}$ \\
\hline$b-y$ & $\begin{array}{r}0.407 \\
\pm 5\end{array}$ & $\begin{array}{r}0.210 \\
\pm 4\end{array}$ & $\begin{array}{r}0.093 \\
\pm 7\end{array}$ \\
\hline$m_{1}$ & $\begin{array}{r}-0.004 \\
\pm 13\end{array}$ & $\begin{array}{r}0.150 \\
\pm 7\end{array}$ & $\begin{array}{r}0.161 \\
\pm 4\end{array}$ \\
\hline$c_{1}$ & $\begin{array}{r}0.778 \\
\pm 11\end{array}$ & $\begin{array}{r}0.917 \\
\pm 11\end{array}$ & $\begin{array}{r}0.943 \\
\pm 2\end{array}$ \\
\hline$\beta$ & $\begin{array}{r}2.783 \\
\pm 1\end{array}$ & $\begin{array}{r}2.849 \\
\pm 3\end{array}$ & $\begin{array}{r}2.865 \\
\pm 1\end{array}$ \\
\hline
\end{tabular}

the applicability of current theoretical models for PMS stars, and understand the variability and unusual photometric phenomena of TY CrA, a careful photometric analysis based on complete and accurate light curves is essential. The observational material for such studies is documented in the present paper, while a detailed analysis of the $y$ light curve (including an ephemeris) and a discussion of the properties of the system are given in Paper III.

The light curves of TY CrA show a moderately deep primary eclipse and a very shallow secondary minimum, 0 . 03 deep in $y$ and barely detectable in $u$. The unequal minima indicate different temperatures for the two components. Anticipating Paper III, the $3.16 M_{\odot}, 12000 \mathrm{~K}$ primary is located near the ZAMS (although curiously cooler than models predict) and the $1.64 M_{\odot}, 4900 \mathrm{~K}$ secondary is at the base of its Hayashi track. A conspicuous "reflection effect" is present, but no other strong proximity effects appear in the light curves. There is evidence of considerable intrinsic variability, on time scales ranging from days to years and not evidently correlated with orbital phase. We discuss the variability further below and suggest that it is primarily the result of variable obscuration.

\section{Observations}

TY CrA was observed in the Strömgren uvby system with the SAT reflecting telescope at ESO, La Silla, Chile, using the six-channel spectrograph-photometer and photon counting system described by Nielsen et al. (1987). Observations were obtained during 22 nights from June 14 to July 9, 1989 (by IH), 5 nights from June 8 to 14 , 1992 (JA), 19 nights from May 26 to June 27, 1993 (LPV, BWC, JA) and 32 nights from June 05 to July 15, 1994
(LPV). A circular diaphragm of $17^{\prime \prime}$ diameter was used throughout.

Sky background measurements were taken systematically at a position $60^{\prime \prime} \mathrm{N}$ and $60^{\prime \prime} \mathrm{E}$ from TYCrA. However, these did not correct for the contribution of the reflection nebulae NGC 6726/7. Correction for the nebular light is discussed in detail in Paper III, where the nebula is found to contribute roughly one third of the observed flux in the $17^{\prime \prime}$ diaphragm at quadrature in all of the colours.

Extinction corrections were based on the nightly coefficients from the two comparison stars and other constant stars. When needed, linear or quadratic corrections for instrumental drift (uncooled photomultipliers) and/or changes in sky transparency during the night, determined from repeated measurement of several constant stars, were applied. HD 176423 and HD 176497 , both within 0.6 of TY CrA, were used as comparison stars and observed alternately between the measurements of TY CrA. Both stars were found to be constant within the observational accuracy throughout the four observing periods. The observational mean errors of a magnitude difference between the comparison stars (data from 1992, 1993 and 1994; the errors in 1989 were roughly a factor 1.2 higher) were:

$\sigma(\Delta u)=0.008, \quad \sigma(\Delta v)=0.007$

$\sigma(\Delta b)=0{ }^{\mathrm{m}} 007, \quad \sigma(\Delta y)=0{ }^{\mathrm{m}} 006$.

Information and photometric results for both TY CrA and the comparison stars are given in Table 1. The photometric indices for TY CrA have not been corrected for nebular contamination. While three stars contribute to the flux from the system, both the primary/secondary and primary/tertiary luminosity ratios are greater than 30 . Thus the photometric indices (once corrected for the nebular contamination, see Paper III) primarily correspond to the photosphere of the primary star.

\section{The light curves}

The light curves of TY CrA in the instrumental system are presented in Tables $2 \mathrm{a}-2 \mathrm{~d}$, containing 698 points from 1989 (Table 2a), 135 points from 1992 (Table 2b), 371 points from 1993 (Table 2c), and 585 points from 1994 (Table 2d) in each colour. Typical rms errors of one magnitude difference (calculated from all points and their deviation from a third order spline interpolation curve used in the calculation of the normal points, see Vaz et al. 1995) are $0.006(u, v)$ and $0.005(b, y)$. Most phases were covered at least twice in the periods 89, 92-93 and 94 .

The $y$ light curves TY CrA - HD 176423 derived from the 1989, 1992 and 1993 observations are shown in Fig. 1, phased according to the ephemeris presented in Paper III: Min I at: HJD $2449537.8414+2.888779 \mathrm{E}$.

$$
\pm 8 \quad \pm 13
$$

As discussed in Paper III, the period is variable, with minima occurring up to 0.004 days from those predicted by 


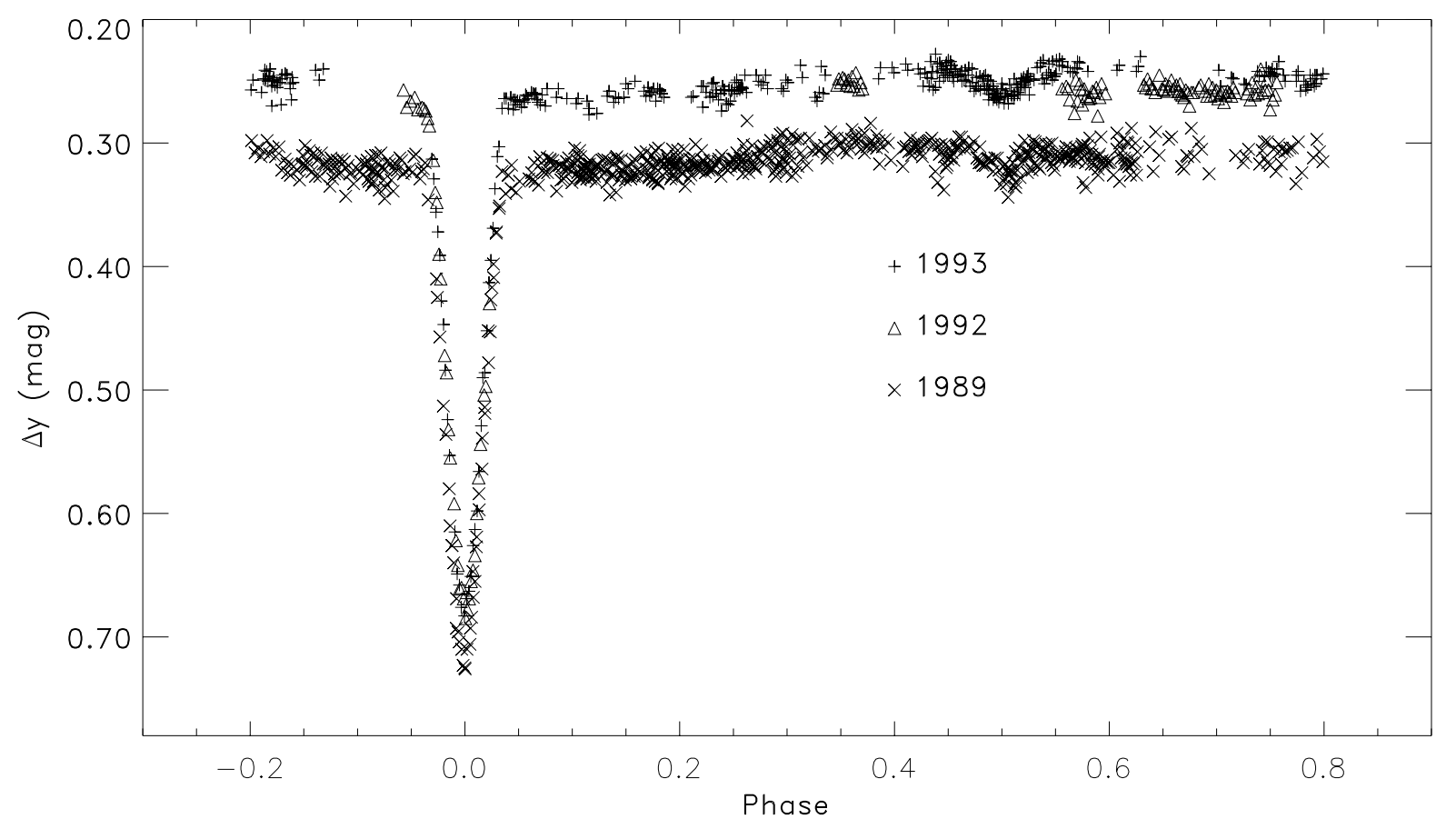

Fig. 1. $y$ magnitude differences (instrumental system) between TY CrA and HD 176423 in 1989, 1992 and 1993

Table 3. Transformation coefficients for calibration of the instrumental system to the standard uvby system, for years 1993 and 1994

\begin{tabular}{|c|c|c|c|c|}
\hline coefficient & \multicolumn{2}{|r|}{1993} & \multicolumn{2}{|c|}{1994} \\
\hline$B$ & 0.061 & \pm 0.032 & $0.032 \pm$ & 0.012 \\
\hline$D$ & 1.022 & \pm 0.008 & $1.028 \pm$ & 0.007 \\
\hline$J$ & 0.019 & \pm 0.015 & $-0.008 \pm$ & 0.014 \\
\hline$F$ & 0.954 & \pm 0.074 & $0.955 \pm$ & 0.053 \\
\hline$I$ & 0.103 & \pm 0.017 & $0.142 \pm$ & 0.025 \\
\hline
\end{tabular}

the above ephemeris. This indicates that orbit of the tertiary star extends some \pm 0.35 AU along the line of sight, possibly more.

The $y$ light curve from 1994 is shown in Fig. 2. The colour index curves from the 1992 and the 1993 data are shown in Fig. 2 of Paper III, and the colour index curves for the 1994 data are shown here in Fig. 3 (the larger uncertainties of the 1989 data do not justify a more detailed presentation of the colours curves). The larger measurement errors in $u$ are apparent in the scatter of the $(u-y)$ index curve.

Coefficients for transformation to the standard system are presented in Table 3, for the 1993 and 1994 observing runs, respectively. They should be used with the instrumental magnitude differences (TY CrA-HD 176423 , Tables 2a-2d) according to the equations (Olsen 1983):

$\Delta V_{\text {std }}=\Delta y_{\text {intr }}+\mathrm{B} \Delta(b-y)_{\text {std }}$

$\Delta(b-y)_{\mathrm{std}}=\mathrm{D} \Delta(b-y)_{\mathrm{instr}}$

$\Delta m_{1, \mathrm{std}}=\mathrm{F} \Delta m_{1, \text { instr }}+\mathrm{J} \Delta(b-y)_{\mathrm{std}}$
$\Delta c_{1, \mathrm{std}}=\mathrm{H} \Delta c_{1, \text { instr }}+\mathrm{I} \Delta(b-y)_{\text {std }}$.

Following Olsen (1983), these transformation coefficients have been determined using standard stars of spectral type $\mathrm{F}$ and earlier, i.e. $(b-y)_{\mathrm{std}}<0.41$, a domain which is valid for both the comparison stars and for TY CrA itself, which is dominated by the light of the early-type primary star.

\section{Non-eclipse variability}

The brightness level of the TY CrA light curve changed significantly during the 5-year period on several time scales. Figure 1 shows that the system became $\sim 0.07$ brighter from 1989 to 1992/1993. The 1994 observations (Fig. 2) show that this tendency subsequently reversed, and the system became again fainter than in 1993 by $\sim 0$. 03 in all four colours (see also Fig. 3 in Paper III).

Non-eclipse variations are also present on much shorter time scales. This is evident in our extensive 1994 data set obtained in an interval covering 42 days ( $~ 14$ orbits); the phased light curve is shown in Fig. 2. Variability is evident at numerous phases where the range of photometry can be as large as 0.05 , far greater than the observational errors. Variability is also evident in the brightnesses of both the shoulders and minima of the primary eclipse. However, we have data from many nights when both the minimum and one of the shoulders of the primary eclipse are observed; in all cases the eclipse depth remains unchanged.

Another perspective on this non-eclipse photometric variations is given in Fig. 4, where we show nightly mean $\mathrm{O}-\mathrm{C}$ values from the theoretical $y$ light curve computed in 


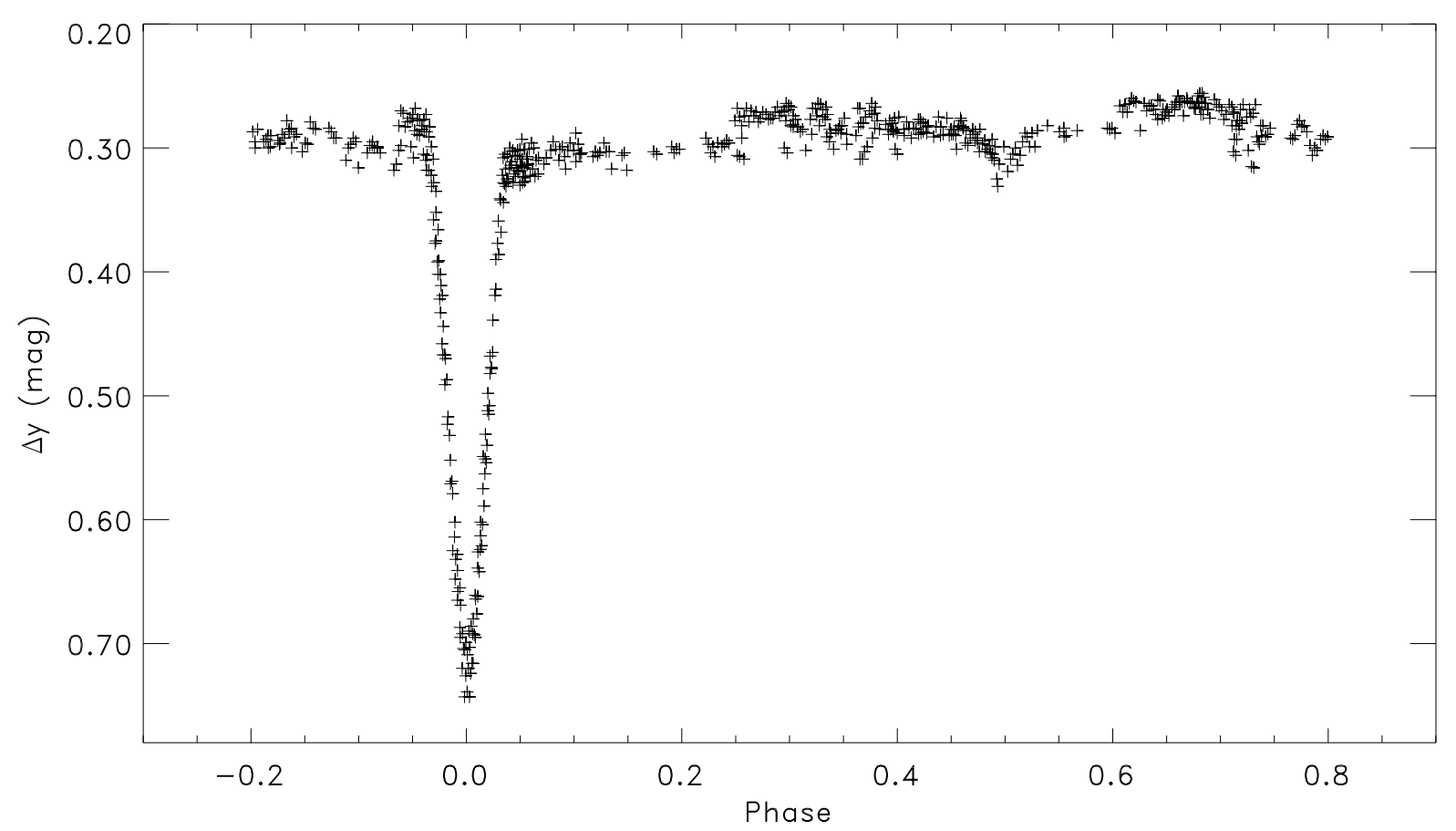

Fig. 2. $y$ magnitude differences (instrumental system) between TY CrA and HD 176423 in 1994

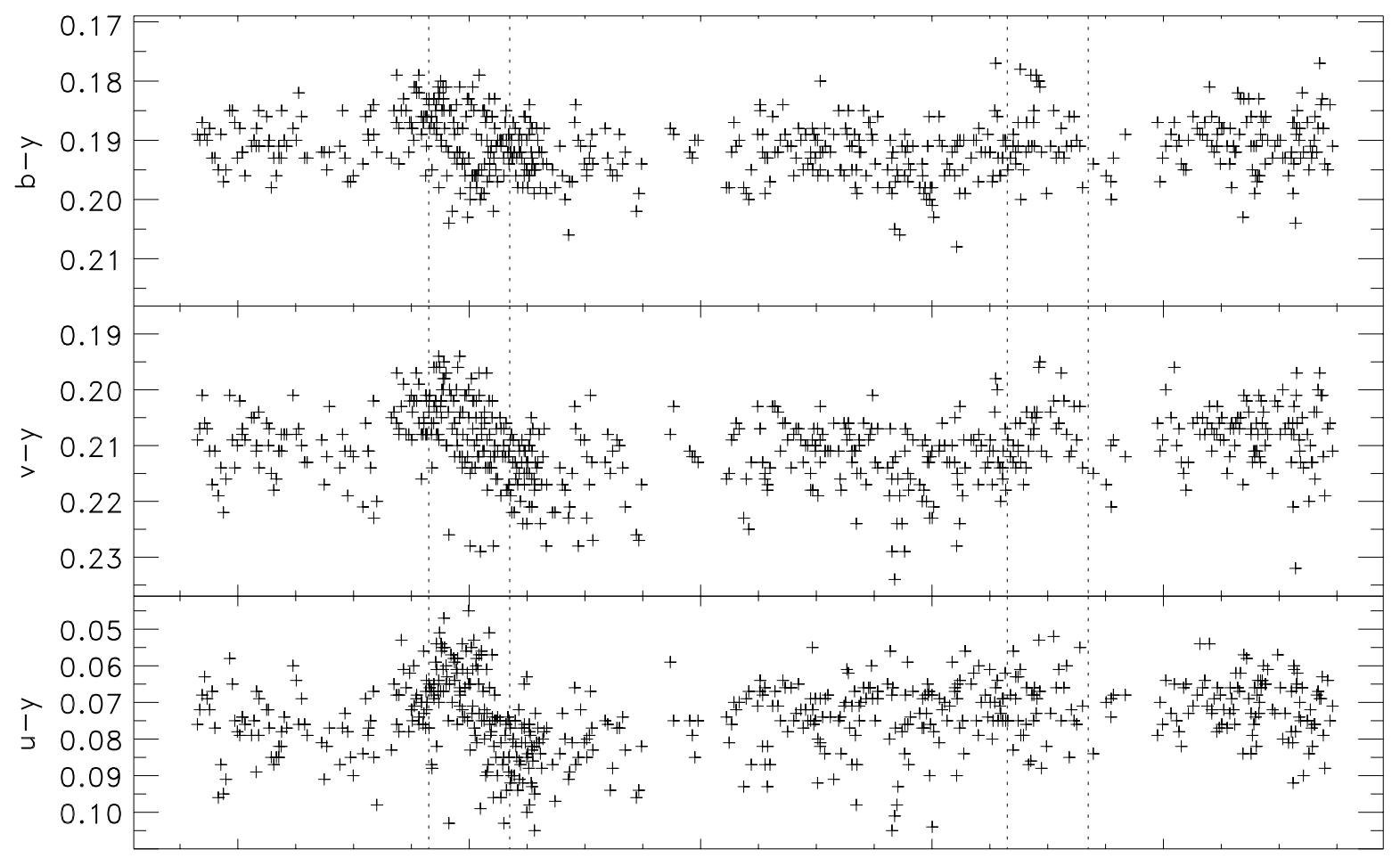

phase

Fig. 3. $b-y, v-y$ and $u-y$ colour index differences (instrumental system) between TY CrA and HD 176423 in 1994 . The vertical dotted lines mark the phase limits of both eclipses, according to the theoretical solution of Paper III 


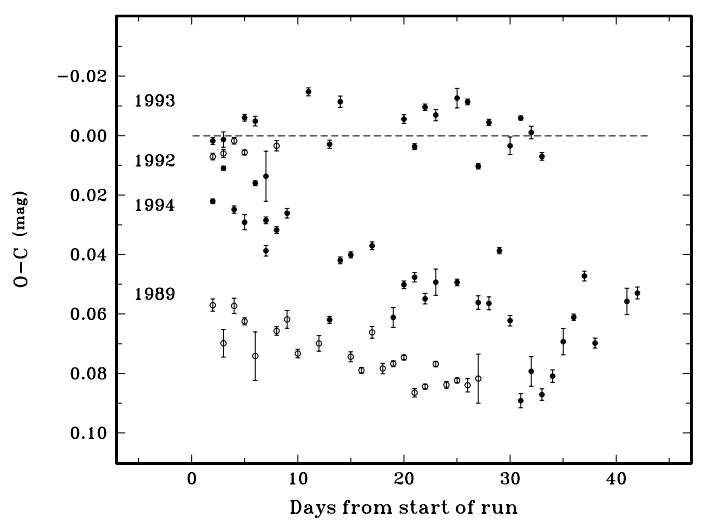

Fig. 4. Nightly mean $\mathrm{O}-\mathrm{C}$ values from the theoretical $y$ light curve (Paper III) plotted with all observing periods referred to the first night so as to have a common origin. The night-tonight variations are clearly seen

Paper III. For the 1994 data there is an evident decrease in the mean light level by $0 . \mathrm{m} 04$ over the course of $30-35$ days, followed by a slight increase over the next week. Evidently non-eclipse variations occurs over the course of a few orbits. Occasional significant variations are also seen on time scales of a day, and thus shorter than the orbital time scale. It is clear from Fig. 4 that, in our measurements covering from 1989 to 1994, TY CrA changed its brightness by $0^{\mathrm{m}} 10$ and reached its brightest levels in 1993 .

As is evident in the colour-index curves (Figs. 3 and 4 in Paper III), these non-eclipse variations are grey to first order. However, more detailed examination reveals a reddening associated with the brightness variations. Extending the calculation of the mean $\mathrm{O}-\mathrm{C}$ to the other (bvu) colours, we show in Fig. 5 the mean $\mathrm{O}-\mathrm{C}$ in $(b-y)$ against the mean $\mathrm{O}-\mathrm{C}$ in $y$. There is a clear trend showing that the fainter the star, the redder its colour.

Given this reddening and the lack of phasing with the orbit, it is tempting to associate these light variations with variable dust obscuration, as also has been demonstrated for the PMS binary AK Sco (Andersen et al. 1989), which can show variations of more than 1 mag in the time scale of one day (later unpublished observations). It seems unlikely that long-term variations as large as 0.05 are intrinsic to the stars in TY CrA. The secondary and tertiary stars individually contribute less than $3 \%$ to the total light, thus making spots on these stars an unlikely origin. Flares are common during the PMS phase of evolution, but they can hardly be the reason for slow variations on time scales of tens of days and longer. An intrinsic variation of the primary star also seems unlikely, considering that the depth of the primary eclipse remains unchanged in the course of the light variations. The same argument applies to varying contributions from the reflection nebula. Barring these explanations, we suggest that at least the longer term variations are due to variable dust obscuration.

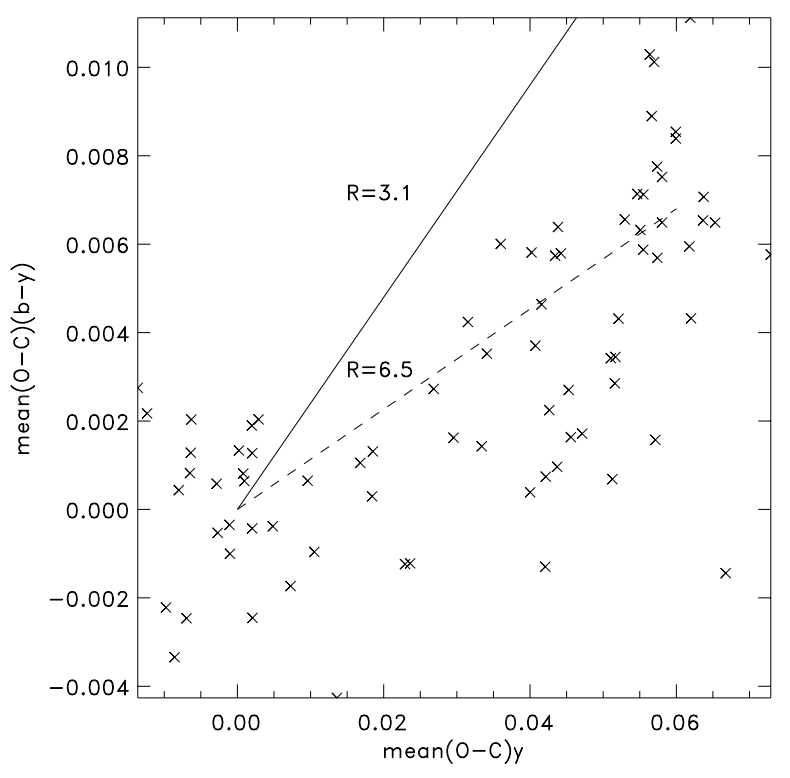

Fig. 5. Colour index curve $\Delta(b-y)$ versus $\Delta y$ for the amount of extinction derived from nightly $\mathrm{O}-\mathrm{C}$ means using the adopted solution of Paper III. Different observing runs are shown with different symbols. The stronger the effect the redder its influence on the light curves. Mean total-to-selective extinction laws (Cardelli et al. 1989) corresponding to $R=3.1$ (continuous line) and to $R=6.5$ (dashed line) are also shown

Figure 5 shows curves orresponding to mean total-toselective extinctions of $R=3.1$ and $R=6.5$, from Cardelli et al. (1989); $R=6.5$ is the value found by Cardelli \& Wallerstein (1989) for TY CrA. Our data are also consistent with such a large value of $R$. Our observations do not allow us to determine the location of the intervening dust. The spectral energy distribution (e.g. Bibo et al. 1992, Paper I) shows a large flux excess longward of $10 \mu \mathrm{m}$, indicating the presence of circumbinary dust. The increasing flux with increasing wavelength suggests a circumbinary shell, plausibly the source of variable extinction. The presence of a circumbinary disk with a central hole cleared of dust is also possible and may contribute to the variability. For an estimated tertiary orbital radius of order $0.5 \mathrm{AU}$, disks might form around both the eclipsing pair and the tertiary star as well as surrounding the entire system, and the tertiary star may be able to influence the dynamics of the dust even if it were not to have a disk of its own. A concerted observational effort will be needed to clarify the dust distribution in the TY CrA system in more detail.

However, not all of the photometric variability is straightforwardly explained by circumbinary obscuration. For example, Paper III notes that during 1992 the system became $0^{\mathrm{m}} 015$ fainter at phase 0.7 in three sequential orbit cycles, strongly suggesting that the variation was linked to orbital phase, although evidently not to the eclipse 
phenomena. Undoubtedly, the TY CrA system harbours more secrets than we have yet been able to unravel.

\section{Colour-index curves}

Examination of the colour index curves reveals the expected bluening in colour during the secondary eclipse (phase 0.5$)$ in the $(b-y)$ and $(v-y)$ curves, a result of decreasing the contribution of the red secondary relative to the blue primary. Note that the primary eclipse also exhibits a bluening in colour. During primary eclipse the secondary star makes a relatively larger contribution to the combined light. However, the nebular contamination also increases relative to the primary. Since the blue nebula contributes more light than the faint, red secondary, the light curve becomes bluer in the primary eclipse.

Another common feature in the colour-index curves of all 4 runs (data from 1994 in Fig. 3; from 1992 and 1993 in Fig. 2 of Paper III) is the small asymmetry in the bluening of the primary minimum, with the first half of the minimum being slightly redder than the second half. The effect was considered too small relative to the observational errors in Paper III, but it is real and present in all colours, even in $y$ and becoming stronger at shorter wavelengths. Since this asymmetry is present in our observations from 1989 to 1994 , it is stable and related to the orbital phase, probably connected to regions of unequal brightness (e.g. spots due to slight but continued accretion) on or near the surface of the eclipsing components.

Finally, the scatter in the 1994 colour curves appears to be significantly reduced during secondary eclipse (see the $b-y$ and $v-y$ colour curves in Fig. 3). A possible explanation is that there is a reduced contribution from surface activity on the secondary star when it is eclipsed. However, as noted above, such activity is unable account for the bulk of the observed non-eclipse variability.

\section{Conclusions}

We present high-precision photometric observations with complete uvby light curves of pre-main sequence eclipsing binary TY CrA, revealing its shallow secondary eclipse. Beyond the variations connected to the orbit (eclipses, reflection effect, etc.), the system presents non-eclipse photometric variations in time scales varying from a fraction of a day to years and which show reddening with decreasing brightness. We suggest that these variations may be linked to dust shells physically associated to the system.

Acknowledgements. This investigation was supported by allocations of ESO and Danish observing time and by grants from the Danish Natural Science Research Council, the Danish Board for Astronomical Research, and the Brazilian institutions CNPq, FAPEMIG, FINEP, CAPES. BWC acknowledges support from the Wisconsin Alumni Research Foundation and the Wisconsin Space Grant Consortium. RDM acknowledges support from a Presidential Young Investigator award and NSF grant AST94-1715. LPV gratefully acknowledges the hospitality received at the Department of Astronomy, University of Wisconsin, Madison, while being a research scholar from Aug 93 to Oct $94(\mathrm{CNPq})$. This research has made use of the Simbad database, operated at CDS, Strasbourg, France.

\section{References}

Andersen J., Lindgren H., Hazen M.L., Mayor M., 1989, A\&A 219, 142

Beust H., Corporon P., Siess L., Forestini M., Lagrange A.-M., 1997, A\&A 320, 478

Bibo E.A., Thé P.S., Dawanas D.N., 1992, A\&A 260293

Cardelli J.A., Wallerstein G., 1989, AJ 97, 1099

Cardelli J.A., Clayton G.C., Mathis J.S., 1989, ApJ 345, 245

Casey B.W., Mathieu R.D., Suntzeff N.B., Lee C.W., Cardelli J.A., 1993, AJ 105, 2276 (Paper I)

Casey B.W., Mathieu R.D., Suntzeff N.B., Walter F.B., 1995, AJ 109, 2156 (Paper II)

Casey B.W., Mathieu R.D., Vaz L.P.R., Andersen J., Suntzeff N.B., 1997, AJ (in press) (Paper III)

Corporon P., Lagrange A.-M., Bouvier J., 1994, A\&A 282, L21 (erratum, A\&A 289, 660, 1994)

Corporon P., Lagrange A.-M., Beust H., 1996, A\&A 310, 228

Durisen R.H., Sterzik M.F., 1994, A\&A 286, 84

Friedemann C., Gürtler J., Löwe M., 1996, A\&AS 117, 205

Haikala L.K., 1994 (private communication)

Harju I.K., Haikala L.K., Mattila K., Mauersberger R., Booth R.S., Nordth H.L., 1993, A\&A 278, 569

Kardopolov V.I., Sahanionok V.V., Philipjev G.K., 1981, Peremennye Zvezdy (Variable Stars) 21, No. 4, 589

Lagrange A.-M., Corporon P., Bouvier J., 1993, A\&A 274, 785

Nielsen R.F., Nørregaard P., Olsen E.H., 1987, ESO Messenger 50,45

Olsen E.H., 1983, A\&AS 54, 55

Proust D., Ochsenbein F., Pettersen B.R., 1981, A\&AS 44, 179

Popper D.M., 1987, ApJ, 313, L81

Vaz L.P.R., Andersen J., Rabello Soares M.C.A., 1995, A\&A 301,693

Vitrichenko E.A., Shevchenko V.S., 1995, Astron. Lett. 21(3), 386 (translated from Pis'ma v. Astronomicheskii Zhurnal $21(6), 434)$ 